\title{
Factor Analysis and Mathematical Modeling in Determining the Quality of Coal
}

\author{
Tomasz NIEDOBA ${ }^{1)}$, Paulina PIETTA2), Agnieszka SUROWIAK ${ }^{1)}$
}

\footnotetext{
1) AGH, University of Science and Technology, Faculty of Mining and Geoengineering, Department of Mineral Processing and Environmental Engineering, Cracow, Poland; email: tniedoba@agh.edu.pl

2) JSW Innowacje S.A.
}

http://doi.org/10.29227/IM-2020-01-24

Submission date: $30-11-2019$ | Review date: 28-03-2020

Abstract
The separation of coal material of three types of coals originating from three various Polish hard coal mines (types 31, 34.2 and 35,
according to Polish nomenclature, which were steam coal, semi-coking coal and coking coal) into particle size fractions and then
into particle density fractions was done and then the following parameters were measured for each particle size-density fraction:
combustion heat, ash contents, sulfur contents, volatile parts contents, analytic moisture. In this way a 7-dimensional vector of data
was created. Using methods of factor analysis the important features of coal were selected, which decide about their membership to
individual types. To evaluate the appropriateness of the applied method the Bartlett's sphericity test as well coefficient of Kaiser-May-
er-Olkin (KMO) were used. To select important factors the Kaiser criterion and Cattell's scree test were used. The obtained results were
compared with the results obtained in previous works by means of observation tunnels method. The results showed which particular
features are crucial to define the type of coal what is also important to select appropriate method of its enrichment. Furthermore, the
construction of a mathematical model presenting the relations between these properties and particle size and density is presented.
Because of the fact that particles of certain size or density may occur in neighboring fractions three sorts of relations were examined
basing on regression analysis. The analysis was conducted for all three coal types. Because of the fact that the models contain various
amounts of independent variables $R^{2}$ coefficient, mean squared error (MSE) and Mallow's statistics Cp were applied to evaluate and
compare obtained results.

Keywords: coal, multidimensional statistical analysis, factor analysis, quality of coal, particle size, particle density

\section{Introduction}

Mineral raw materials which are beneficiated in purpose of their using characterize with many factors describing their features. In case of coal, these features are among others ash contents, sulfur contents, combustion heat, volatile parts contents or analytic moisture. The features mentioned above decide about coal quality also in economical aspect. Because of that the preciseness of determining values of these features is very important.

The most often researched properties of the coal are combustion heat, ash contents, sulphur contents, volatile parts contents and moisture. These features are very often highly correlated but also can occur independently. The selection of the necessary factors which influence on individual properties is the goal of the paper. To this purpose three types of coal (according to Polish nomenclature - coal types 31 (steam coal), 34.2 (gas-coking coal) and 35 (orto-coking coal)) were selected to the investigation which were divided into particle size and density fractions. The classification of coals is presented in Table 1.

The whole group of considered factors were measured for each size-density fraction [14].

The following variables were considered $\left(X_{i}=1,2, \ldots, 5\right)$.

$\mathrm{X}_{1}$ - combustion heat [cal];

$\mathrm{X}_{2}$ - ash contents [\%];

$\mathrm{X}_{3}$ - sulfur contents [\%];

$\mathrm{X}_{4}$ - volatile parts contents [Va];

$\mathrm{X}_{5}-$ moisture $[\mathrm{Wa}]$.
Knowledge about these features can serve also to evaluate beneficiation process (Brożek, 1984; Dobosz, 2001; Foszcz et al., 2016; Głowiak 2019a; b; Niedoba, 2013a; Stanisz, 2007; Stępiński, 1964; Tumidajski and Saramak, 2009). The ash contents, sulfur contents and volatile parts contents were investigated dependably on particle size and particle density also by means of kriging method (Niedoba, 2013a). The application of non-conventional statistical methods can be very beneficial in getting precise information (Foszcz et al., 2018; Jamróz, 2009; 2014a; b; c; Jamróz et al., 2016; 2017; Jamróz and Niedoba, 2014; 2015a; b; Niedoba, 2009; 2011; 2013a; b; 2014; 2015; Niedoba and Surowiak, 2012; Niedoba et al., 2018; Öney, 2019; Pięta et al., 2018; Surowiak 2007, 2014). The presented work is an attempt of constructing new mathematical model describing relation between ash contents and particle size and density.

\section{Materials and methods}

The considered types of coal originated from three various Polish coal mines and all of them were initially screened on a set of sieves of the following sizes: $-1.00,-3.15,-6.30,-8.00$, $-10.00,-12,50,-14.00,-16.00$ and $-20.00 \mathrm{~mm}$. Then, the size fractions were additionally separated into density fractions by separation in dense media using zinc chloride aqueous solution of various densities $(1.3,1.4,1.5,1.6,1.7,1.8$ and $1.9 \mathrm{~g} /$ $\mathrm{cm}^{3}$ ). The fractions were used as a basis for further consideration and additional coal features were determined by means of chemical analysis. In purpose of appropriate identification 
Tab. 1. Classification of coal types according to Polish nomenclature (Sobolewski et al., 2016)

Tab. 1. Klasyfikacja węgli według polskiej nomenklatury (Sobolewski et al., 2016)

\begin{tabular}{|l|c|c|}
\hline Coal type & Coal number & Volatile parts contents [\%] \\
\hline Steam coal & 31 & Above 28 \\
\hline Gas-steam coal & 32 & Above 28 \\
\hline Gas coal & 33 & Above 28 \\
\hline Gas-coking coal & 34 & Above 28 \\
\hline Orto-coking coal & 35 & $20-31$ \\
\hline Meta-coking coal & 36 & $14-28$ \\
\hline Semi-coking coal & 37 & $14-28$ \\
\hline Thin coal & 38 & $14-28$ \\
\hline Anthracite coal & 41 & $10-14$ \\
\hline Anthracite & 42 & $3-10$ \\
\hline Meta-anthracite & 43 & Till 3 \\
\hline
\end{tabular}

Tab. 2. Data for size fraction 14.00-12.50 $\mathrm{mm}$ - coal, type 31

Tab. 2. Dane dla klasy ziarnowej 14.00-12.50 - węgiel, typ 31

\begin{tabular}{|c|c|c|c|c|c|c|}
\hline $\begin{array}{c}\text { Density } \\
{\left[\mathbf{M g} / \mathbf{m}^{\mathbf{3}} \mathbf{]}\right.}\end{array}$ & $\begin{array}{c}\text { Mass } \\
\mathbf{[ g ]}\end{array}$ & $\begin{array}{c}\text { Combustion heat } \\
{[\mathbf{c a l}]}\end{array}$ & $\begin{array}{c}\text { Ash } \\
\mathbf{c o n t e n t s} \\
{[\mathbf{[ \% ]}}\end{array}$ & $\begin{array}{c}\text { Sulfur } \\
\text { contents [\%] }\end{array}$ & $\begin{array}{c}\text { Volatile parts } \\
\text { contents } \\
\mathbf{V}^{\mathbf{a}}\end{array}$ & $\begin{array}{c}\text { Analytical } \\
\text { moisture } \mathbf{W}_{\mathbf{a}}\end{array}$ \\
\hline$<1.3$ & 308.6 & 7048 & 6.41 & 0.72 & 34.32 & 3.23 \\
\hline $1.3-1.4$ & 292.5 & 5859 & 19.61 & 0.7 & 29.22 & 3.36 \\
\hline $1.4-1.5$ & 36.1 & 2948 & 16.55 & 0.76 & 28.92 & 3.87 \\
\hline $1.5-1.6$ & 10.7 & 5117 & 26.10 & 1.55 & 31.08 & 3.40 \\
\hline $1.6-1.7$ & 25.6 & 4467 & 35.78 & 2.28 & 26.71 & 2.40 \\
\hline $1.7-1.8$ & 139 & 3920 & 37.20 & 1.23 & 29.24 & 2.19 \\
\hline $1.8-1.9$ & 12.7 & 3078 & 48.20 & 1.13 & 24.05 & 2.23 \\
\hline$>1.9$ & 601.2 & 457 & 86.53 & 0.40 & 9.30 & 0.91 \\
\hline
\end{tabular}

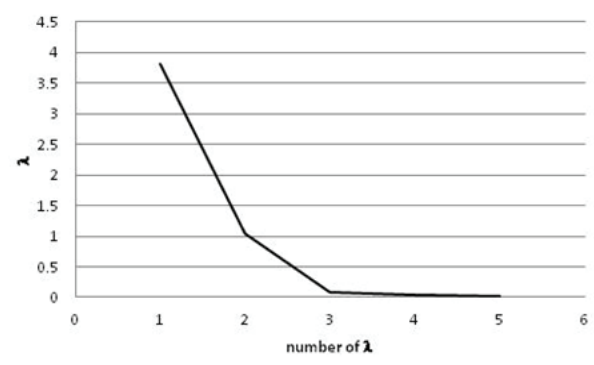

Fig. 1. Cattell's scree plot for coal, type 31, particle size fraction (10-12.5) Rys. 1. Wykres osypiska Cattella dla węgla, typ 31, klasa ziarnowa (10-12.5)

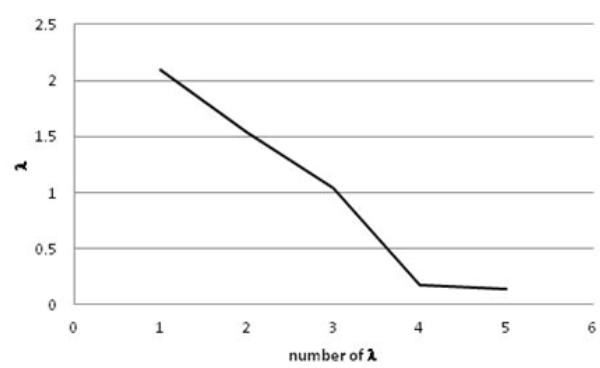

Fig. 2. Cattell's scree plot for coal, type 34.2, particle density fraction (1.6-1.7) Rys. 2. Wykres osypiska Cattella dla węgla, typ 34.2, frakcja gęstościowa (1.6-1.7)

of coal type many parameters are being measured which describe coal quality. For each density-size fraction such parameters as combustion heat, ash contents, sulfur contents, volatile parts contents and analytical moisture were determined, making up, together with the mass of these fractions, seven various features for each coal.

The example of obtained data is presented in Table 2.

The measurements of $\mathrm{X}_{\mathrm{i}}$ were performed for each size-density fraction. Because of the fact that the individual features were measured in various units their standardization was done.
In purpose of selecting significant factors influencing on individual variables, the factor analysis method was applied. To evaluate adequacy of applying factor analysis to this problem two criteria were used: Bartlett's test and Kaiser-Mayer-Olkin coefficient (KMO) (Comrey, 1973; Dobosz, 2001; Kline, 1994;Lawley and Maxwell, 1971; Tumidajski and Saramak, 2009).

The reduction of variables is done through the Cattell's scree criteria and criterion of sufficient proportion which suggest to apply such number of factors that they explain together at least $85 \%$ of variance of all observed variables [Stanisz, 2007]. 
Tab. 3. Influence of factors on properties of coal, type 31 by particle size fractions Tab. 3. Wpływ czynników na właściwości węgla, typ 31 według klas ziarnowych

\begin{tabular}{|c|c|c|c|c|c|c|c|c|c|}
\hline Feature & $0.5-1$ & $1-3.15$ & $3.15-6.3$ & 6.3-8 & 8-10 & $10-12.5$ & $12.5-14$ & 14-16 & $16-20$ \\
\hline \multicolumn{10}{|c|}{ Combustion heat } \\
\hline$Z_{1}$ & 97.49 & 95.39 & 97.57 & 97.63 & 94.13 & 96.29 & 81.52 & 95.41 & 99.89 \\
\hline$Z_{2}$ & & & & & & & 11.42 & & \\
\hline \multicolumn{10}{|c|}{ Ash contents } \\
\hline$Z_{1}$ & 86.47 & 95.55 & 90.68 & 99.34 & 95.86 & 96.58 & 97.33 & 97.65 & 99.28 \\
\hline \multicolumn{10}{|c|}{ Sulfur contents } \\
\hline$Z_{1}$ & 95.74 & 68.65 & 94.59 & 7.99 & 5.00 & 0.04 & 2.31 & 22.45 & 2.06 \\
\hline$Z_{2}$ & & 30.83 & & 91.96 & 94.63 & 99.26 & 90.13 & 77.28 & 97.19 \\
\hline \multicolumn{10}{|c|}{ Volatile parts contents } \\
\hline$Z_{1}$ & 94.71 & 95.60 & 84.65 & 88.34 & 93.41 & 95.90 & 96.09 & 88.56 & 86.71 \\
\hline$Z_{2}$ & & & 12.11 & & & & & & \\
\hline \multicolumn{10}{|c|}{ Moisture } \\
\hline$Z_{1}$ & 93.25 & 95.21 & 75.25 & 95.19 & 95.90 & 92.34 & 79.90 & 97.35 & 94.22 \\
\hline$Z_{2}$ & & & 18.16 & & & & 7.00 & & \\
\hline
\end{tabular}

Tab. 4. Influence of factors on properties of coal, type 34.2 by particle size fractions Tab. 4. Wpływ czynników na właściwości węgla, typ 34.2 według klas ziarnowych

\begin{tabular}{|c|c|c|c|c|c|c|c|c|c|}
\hline Feature & $0.5-1$ & $1-3.15$ & $3.15-6.3$ & 6.3-8 & $8-10$ & $10-12.5$ & $12.5-14$ & 14-16 & $16-20$ \\
\hline \multicolumn{10}{|c|}{ Combustion heat } \\
\hline$Z_{1}$ & 97.89 & 97.73 & 88.58 & 65.37 & 94.14 & 86.65 & 82.95 & 95.90 & 91.83 \\
\hline$Z_{2}$ & & & & 6.96 & & & & & \\
\hline$Z_{3}$ & & & & 27.60 & & & & & \\
\hline \multicolumn{10}{|c|}{ Ash contents } \\
\hline$Z_{1}$ & 97.37 & 96.35 & 99.52 & 93.33 & 94.53 & 91.83 & 87.25 & 91.83 & 85.34 \\
\hline \multicolumn{10}{|c|}{ Sulfur contents } \\
\hline$Z_{1}$ & 97.51 & 62.60 & 1.84 & 9.92 & 31.49 & 54.43 & 20.79 & 6.25 & 52.26 \\
\hline$Z_{2}$ & & 16.74 & 95.29 & 88.37 & 65.15 & 11.59 & 72.53 & 91.43 & 44.28 \\
\hline$Z_{3}$ & & 5.85 & & & & 31.21 & & & \\
\hline \multicolumn{10}{|c|}{ Volatile parts contents } \\
\hline$Z_{1}$ & 61.29 & 86.22 & 92.58 & 93.91 & 90.68 & 77.51 & 68.14 & 84.89 & 82.95 \\
\hline$Z_{2}$ & 25.35 & & & & & 11.59 & 26.65 & 0.09 & 14.36 \\
\hline$Z_{3}$ & & & & & & & & 7.59 & \\
\hline \multicolumn{10}{|c|}{ Moisture } \\
\hline$Z_{1}$ & 22.21 & 49.29 & 30.33 & 89.20 & 70.39 & 24.35 & 38.26 & 64.02 & 66.19 \\
\hline$Z_{2}$ & 73.23 & 32.06 & 3.01 & & 16.54 & 69.87 & 49.75 & 8.34 & 29.99 \\
\hline$Z_{3}$ & & 4.23 & 66.48 & & & & & 21.64 & \\
\hline
\end{tabular}

Tab. 5. Influence of factors on properties of coal, type 35 by particle size fractions Tab. 5. Wpływ czynników na właściwości węgla, typ 35 według klas ziarnowych

\begin{tabular}{|c|c|c|c|c|c|c|c|c|c|}
\hline Feature & $0.5-1$ & $1-3.15$ & $3.15-6.3$ & 6.3-8 & $8-10$ & $10-12.5$ & $12.5-14$ & $14-16$ & $16-20$ \\
\hline \multicolumn{10}{|c|}{ Combustion heat } \\
\hline$Z_{1}$ & 92.23 & 98.46 & 96.29 & 92.87 & 90.70 & 97.12 & 97.49 & 92.14 & 84.89 \\
\hline$Z_{2}$ & & & & & & & & & 13.80 \\
\hline \multicolumn{10}{|c|}{ Ash contents } \\
\hline$Z_{1}$ & 92.23 & 98.01 & 76.47 & 95.29 & 91.75 & 96.80 & 96.68 & 93.89 & 95.08 \\
\hline$Z_{2}$ & & & 12.64 & & & & & & \\
\hline \multicolumn{10}{|c|}{ Sulfur contents } \\
\hline$Z_{1}$ & 12.18 & 4.59 & 6.48 & 1.16 & 24.70 & 2.23 & 0.02 & 4.32 & 26.79 \\
\hline$Z_{2}$ & 70.66 & 77.03 & 34.07 & 97.02 & 52.67 & 69.80 & 67.99 & 75.00 & 62.85 \\
\hline$Z_{3}$ & 15.35 & 18.35 & 48.24 & & 21.51 & 27.23 & 31.84 & 16.42 & \\
\hline \multicolumn{10}{|c|}{ Volatile parts contents } \\
\hline$Z_{1}$ & 78.35 & 87.90 & 99.64 & 92.14 & 76.51 & 92.23 & 84.54 & 87.60 & 87.19 \\
\hline$Z_{2}$ & 6.88 & & & & 13.64 & & 8.93 & & \\
\hline \multicolumn{10}{|c|}{ Moisture } \\
\hline$Z_{1}$ & 15.14 & 36.10 & 54.49 & 40.60 & 44.32 & 0.04 & 2.74 & 1.15 & 15.94 \\
\hline$Z_{2}$ & 68.49 & 29.89 & 31.16 & 13.81 & 46.37 & 89.85 & 63.66 & 83.50 & 72.35 \\
\hline$Z_{3}$ & 14.06 & 26.68 & & 43.74 & & & 33.50 & 15.26 & \\
\hline
\end{tabular}

\section{Results}

\subsection{Factor analysis}

Applying Bartlett's test it occurred that for all researched cases the value of the test was significantly higher than the critical values on significance level being equal to $\alpha=0.0005$. The lowest value of the test $U$ was obtained for coal, type 35 in particle density fraction (1.9-2.0) and was equal to 84.74 , while the critical value on this level is equal to 31.42 . It can 
Tab. 6. Influence of factors on properties of coal, type 31 by particle density fractions Tab. 6. Wpływ czynników na właściwości węgla, typ 31 według frakcji gęstościowych

\begin{tabular}{|c|c|c|c|c|c|c|c|c|}
\hline Feature & $<1.3$ & 1.3-1.4 & $1.4-1.5$ & $1.5-1.6$ & $1.6-1.7$ & $1.7-1.8$ & 1.8-1.9 & $1.9-2.0$ \\
\hline \multicolumn{9}{|c|}{ Combustion heat } \\
\hline$Z_{1}$ & 86.99 & 87.47 & 35.10 & 84.97 & 59.66 & 28.46 & 87.94 & 75.69 \\
\hline$Z_{2}$ & & & 60.40 & 8.15 & 20.53 & 63.98 & & 27.82 \\
\hline$Z_{3}$ & & & & & 9.15 & & & \\
\hline \multicolumn{9}{|c|}{ Ash contents } \\
\hline$Z_{1}$ & 92.42 & 94.03 & 82.88 & 70.94 & 83.86 & 82.04 & 75.15 & 51.62 \\
\hline$Z_{2}$ & & & 1.33 & 25.38 & 11.19 & 0.02 & 9.01 & 36.33 \\
\hline$Z_{3}$ & & & 3.12 & & & 17.05 & 0.01 & \\
\hline$Z_{4}$ & & & & & & & 15.30 & \\
\hline \multicolumn{9}{|c|}{ Sulfur contents } \\
\hline$Z_{1}$ & 7.82 & 17.61 & 35.58 & 64.03 & 36.52 & 18.13 & 1.60 & 40.24 \\
\hline$Z_{2}$ & 56.73 & 80.64 & 48.87 & 4.86 & 36.97 & 67.04 & 87.51 & 54.30 \\
\hline$Z_{3}$ & 34.85 & & 14.49 & 17.92 & 23.87 & & & \\
\hline \multicolumn{9}{|c|}{ Volatile parts contents } \\
\hline$Z_{1}$ & 89.88 & 87.01 & 73.80 & 68.22 & 21.16 & 71.84 & 18.36 & 74.33 \\
\hline$Z_{2}$ & & & 0.03 & 1.26 & 43.08 & 13.03 & 42.04 & 16.73 \\
\hline$Z_{3}$ & & & 0.06 & 6.83 & 35.45 & 1.06 & 38.69 & \\
\hline$Z_{4}$ & & & 24.75 & 23.66 & & & & \\
\hline \multicolumn{9}{|c|}{ Moisture } \\
\hline$Z_{1}$ & 1.87 & 79.85 & 63.42 & 46.36 & 93.10 & 60.04 & 39.66 & 66.11 \\
\hline$Z_{2}$ & 67.24 & 0.09 & 5.97 & 37.93 & & 5.84 & 34.95 & 0.07 \\
\hline$Z_{3}$ & 30.74 & 18.61 & 27.06 & 15.37 & & 31.75 & 0.06 & 37.06 \\
\hline$Z_{4}$ & & & & & & & 24.35 & \\
\hline
\end{tabular}

Tab. 7. Influence of factors on properties of coal, type 34.2 by particle density fractions Tab. 7. Wpływ czynników na właściwości węgla, typ 34.2 według frakcji gęstościowych

\begin{tabular}{|c|c|c|c|c|c|c|c|c|}
\hline Feature & $<\mathbf{1 . 3}$ & $1.3-1.4$ & $1.4-1.5$ & $1.5-1.6$ & 1.6-1.7 & $1.7-1.8$ & $1.8-1.9$ & $1.9-2.0$ \\
\hline \multicolumn{9}{|c|}{ Combustion heat } \\
\hline$Z_{1}$ & 68.90 & 21.37 & 75.81 & 52.56 & 73.37 & 99.60 & 91.83 & 83.37 \\
\hline$Z_{2}$ & 13.54 & 70.82 & 1.03 & 28.72 & 17.60 & & & 7.68 \\
\hline$Z_{3}$ & 14.35 & & 20.48 & 13.13 & & & & \\
\hline \multicolumn{9}{|c|}{ Ash contents } \\
\hline$Z_{1}$ & 80.94 & 83.39 & 89.18 & 73.41 & 34.26 & 13.14 & 87.25 & 6.51 \\
\hline$Z_{2}$ & 6.02 & 10.66 & & 3.69 & 43.21 & 23.72 & & 86.19 \\
\hline$Z_{3}$ & 18.01 & 4.79 & & 9.90 & 14.92 & 46.22 & & \\
\hline$Z_{4}$ & & & & & & 5.34 & & \\
\hline \multicolumn{9}{|c|}{ Sulfur contents } \\
\hline$Z_{1}$ & 18.36 & 83.26 & 63.64 & 53.96 & 30.73 & 51.60 & 37.05 & 95.39 \\
\hline$Z_{2}$ & 64.78 & 11.15 & 8.70 & 0.06 & 56.11 & 48.00 & 52.91 & \\
\hline$Z_{3}$ & 16.30 & & 23.41 & 31.76 & & & & \\
\hline \multicolumn{9}{|c|}{ Volatile parts contents } \\
\hline$Z_{1}$ & 82.88 & 71.58 & 56.23 & 11.26 & 52.25 & 1.46 & 75.65 & 87.32 \\
\hline$Z_{2}$ & 15.10 & 0.06 & 0.01 & 81.28 & 34.98 & 72.72 & 19.64 & \\
\hline$Z_{3}$ & & 26.70 & 34.32 & & & 14.75 & & \\
\hline \multicolumn{9}{|c|}{ Moisture } \\
\hline$Z_{1}$ & 49.75 & 47.22 & 2.97 & 43.19 & 19.22 & 43.02 & 40.90 & 64.67 \\
\hline$Z_{2}$ & 25.45 & 42.22 & 86.52 & 8.72 & 2.11 & 32.11 & 48.26 & 24.86 \\
\hline$Z_{3}$ & 20.63 & & & 39.77 & 76.65 & 4.38 & & \\
\hline$Z_{4}$ & & & & & & 20.13 & & \\
\hline
\end{tabular}

be said then that zero hypothesis (that correlation matrix is a unit matrix) should be rejected for all particle size and density fractions.

Furthermore, it can be noticed that in almost all cases the value of KMO coefficient was higher than 0.5 . Only for density fraction lower than $1.3 \mathrm{~g} / \mathrm{cm}^{3}$ for coal, type 34.2 and density fraction (1.6-1.7) for coal, type 35 it occurred to be slightly lower than 0.5 . That means that the results of Bartlett's test and the values of KMO coefficient gave strong basis to apply factor analysis.
In the work, the reduction of variables is done through the Cattell's scree criteria and criterion of sufficient proportion which suggest to apply such number of factors that they explain together at least $85 \%$ of variance of all observed variables [22].

The correlation matrix of the factor $Z_{j}$ with variable $X_{i}$ is obtained by creation of matrix $Z$, which elements are numbers

$z_{i j}=\sqrt{\lambda_{j} a_{j i}}, \quad i, j=1,2, \ldots, 5$. 
Tab. 8. Influence of factors on properties of coal, type 35 by particle density fractions Tab. 8. Wpływ czynników na właściwości węgla, typ 35 według frakcji gęstościowych

\begin{tabular}{|c|c|c|c|c|c|c|c|c|}
\hline Feature & $<1.3$ & $1.3-1.4$ & $1.4-1.5$ & $1.5-1.6$ & $1.6-1.7$ & $1.7-1.8$ & 1.8-1.9 & $1.9-2.0$ \\
\hline \multicolumn{9}{|c|}{ Combustion heat } \\
\hline$Z_{1}$ & 36.22 & 94.80 & 58.46 & 93.14 & 55.65 & 88.39 & 59.42 & 99.70 \\
\hline$Z_{2}$ & 47.32 & & 0.17 & & 43.09 & & 18.46 & \\
\hline$Z_{3}$ & 13.34 & & 37.14 & & & & 8.12 & \\
\hline \multicolumn{9}{|c|}{ Ash contents } \\
\hline$Z_{1}$ & 36.62 & 97.91 & 15.03 & 94.01 & 71.14 & 76.54 & 77.59 & 59.87 \\
\hline$Z_{2}$ & 47.32 & & 56.07 & & 23.93 & 9.48 & 5.65 & 32.02 \\
\hline$Z_{3}$ & 13.34 & & 27.41 & & & & 3.76 & \\
\hline \multicolumn{9}{|c|}{ Sulfur contents } \\
\hline$Z_{1}$ & 22.05 & 40.51 & 16.48 & 9.04 & 21.90 & 75.06 & 27.98 & 57.54 \\
\hline$Z_{2}$ & 70.30 & 54.61 & 37.22 & 78.17 & 16.54 & 10.15 & 53.01 & 26.44 \\
\hline$Z_{3}$ & & & 17.61 & 7.79 & 54.39 & & & 0.02 \\
\hline$Z_{4}$ & & & 25.56 & & & & & \\
\hline \multicolumn{9}{|c|}{ Volatile parts contents } \\
\hline$Z_{1}$ & 94.78 & 55.74 & 4.00 & 49.97 & 28.64 & 39.06 & 31.14 & 26.50 \\
\hline$Z_{2}$ & & 31.34 & 42.35 & 19.51 & 44.55 & 35.14 & 10.68 & 44.03 \\
\hline$Z_{3}$ & & & 20.81 & 15.70 & 11.12 & 13.42 & 51.94 & 12.22 \\
\hline$Z_{4}$ & & & 31.70 & & 4.93 & & & 9.95 \\
\hline \multicolumn{9}{|c|}{ Moisture } \\
\hline$Z_{1}$ & 70.12 & 23.27 & 86.19 & 7.81 & 27.96 & 5.03 & 52.51 & 6.35 \\
\hline$Z_{2}$ & 14.68 & 1.38 & & 33.79 & 44.98 & 68.92 & 18.25 & 0.21 \\
\hline$Z_{3}$ & 11.48 & 72.67 & & 48.87 & 20.76 & 25.99 & 1.18 & 89.98 \\
\hline$Z_{4}$ & & & & & & & 17.51 & \\
\hline
\end{tabular}

Tab. 9. Ash contents by separation in accordance to particle size - coal, type 31

Tab. 9. Zawartość popiołu według rozdziału na klasy ziarnowe - węgiel, typ 31

\begin{tabular}{|c|c|c|c|c|c|c|c|}
\hline $\boldsymbol{x}_{\mathbf{1}}[\mathbf{m m}]$ & $\boldsymbol{y}[\boldsymbol{\%}]$ & $\boldsymbol{x}_{\mathbf{2}}[\mathbf{\%}]$ & $\boldsymbol{x}_{\mathbf{3}}[\boldsymbol{\%}]$ & $\boldsymbol{y}_{\mathbf{1}}[\mathbf{\%}]$ & $\boldsymbol{y}_{\mathbf{2}}[\boldsymbol{\%}]$ & $\boldsymbol{y}_{\mathbf{3}}[\boldsymbol{\%}]$ & $\boldsymbol{y}_{\mathbf{4}}[\boldsymbol{\%}]$ \\
\hline 0.750 & 12.480 & 0.000 & 13.550 & 12.790 & 11.180 & 10.530 & 10.170 \\
\hline 2.075 & 13.550 & 12.480 & 15.740 & 16.110 & 17.840 & 14.080 & 15.020 \\
\hline 4.725 & 15.740 & 13.550 & 32.000 & 22.740 & 21.950 & 23.150 & 22.750 \\
\hline 7.150 & 32.000 & 15.740 & 41.940 & 28.820 & 26.190 & 30.640 & 29.360 \\
\hline 9.000 & 41.940 & 32.000 & 42.240 & 33.460 & 35.040 & 35.140 & 35.560 \\
\hline 11.250 & 42.240 & 41.940 & 46.150 & 39.090 & 42.020 & 41.200 & 42.100 \\
\hline 13.250 & 46.150 & 42.240 & 47.480 & 44.100 & 44.930 & 46.240 & 46.290 \\
\hline 15.000 & 47.480 & 46.150 & 50.010 & 48.480 & 48.870 & 50.860 & 50.720 \\
\hline 18.000 & 50.010 & 47.480 & 0.000 & 56.000 & 53.580 & 49.800 & 49.610 \\
\hline
\end{tabular}

where: $\lambda_{i}-i^{\text {th }}$ eigenvalue of correlation matrix; $a_{j i}$ - elements of matrix $A$ which fulfills the condition $A^{T}=R$, where $R$ is correlation matrix of variables $X_{j}$.

The square of number $z_{i j}$ is the percentage of variance changeability explained by the factor $Z_{j}$. For example, considering coal, type 31 from the particle size fraction (10-12.5) it is obtained that matrix $Z$ is in form

$Z=\left[\begin{array}{rrcrr}-0.9813 & 0.1331 & -0.0962 & 0.0676 & 0.0747 \\ 0.9828 & -0.1017 & 0.1145 & -0.0767 & 0.0700 \\ -0.0667 & -0.9963 & -0.0484 & 0.0246 & 0.0033 \\ -0.9793 & -0.0651 & -0.0297 & 0.1893 & -0.0019 \\ -0.9620 & -0.1035 & 0.2487 & 0.0063 & -0.0029\end{array}\right]$

The eigenvalues of the correlation matrix are in this case numbers $\lambda_{1}=3.8177 ; \lambda_{2}=1.0355 ; \lambda_{3}=0.0875 ; \lambda_{4}=0.0488$; $\lambda_{5}=0.0105$.

The plot of scree is presented on Figure 1.

On the basis of the presented Cattell's scree plot only these factors remain which are located to the left from the point in which a mild decline of eigenvalues is observed. In this case these are factors $Z_{1}$ and $Z_{2}$.

The group of factors $\left(Z_{1}, Z_{2}\right)$ explain $98.07 \%$ of changeability of combustion heat, $97.12 \%$ of changeability of ash contents, $99.71 \%$ of changeability of sulfur contents, $96.33 \%$ of changeability of volatile parts contents and $93.62 \%$ of changeability of moisture.

It is obtained then that factor $Z_{1}$ is responsible for variables $\left\{X_{1}, X_{2}, X_{4}, X_{5}\right\}$ and factor $Z_{2}$ for variable $X_{3}$.

Let consider the particle density fraction (1.6-1.7) of coal, type 34.2 .

The matrix $\mathrm{Z}$ is in form

$$
\mathrm{Z}=\left[\begin{array}{ccccc}
0.8566 & -0.4196 & -0.1506 & -0.1824 & 0.3643 \\
-0.5854 & -0.6574 & 0.3863 & 0.1622 & 0.1400 \\
0.5544 & -0.7491 & -0.2733 & 0.1882 & -0.2073 \\
-0.7229 & -0.5915 & -0.1757 & -0.2615 & -0.0447 \\
-0.4385 & 0.1454 & -0.8755 & 0.0956 & 0.0961
\end{array}\right]
$$

The eigenvalues of correlation matrix in this case are numbers $\lambda_{1}=2.0993 ; \lambda_{2}=1.5404 ; \lambda_{3}=1.0443 ; \lambda_{4}=0.1727 ; \lambda_{5}=0.1433$. The plot of Cattell's scree is presented on Figure 2.

The Cattell's scree plot suggests to take factors $Z_{1}, Z_{2}$ and $Z_{3}$ into consideration. The same factors explain sufficient percentage of changeability of all observed variables. Group of factors $\left(Z_{1}, Z_{2}, Z_{3}\right)$ explains $93.25 \%$ of changeability of combustion heat, $92.41 \%$ of ash contents, $94.32 \%$ of sulfur contents, $90.33 \%$ of volatile parts contents and $97.99 \%$ of mois- 
Tab. 10. Ash contents by separation in accordance to particle density - coal, type 31

Tab. 10. Zawartość popiołu według rozdziału na frakcje gęstościowe - węgiel, typ 31

\begin{tabular}{|c|c|c|c|c|c|c|c|}
\hline $\boldsymbol{x}_{\mathbf{1}}[\mathbf{m m}]$ & $\boldsymbol{y}[\mathbf{\%}]$ & $\boldsymbol{x}_{\mathbf{2}}[\mathbf{\%}]$ & $\boldsymbol{x}_{\mathbf{3}}[\boldsymbol{\%}]$ & $\boldsymbol{y}_{\mathbf{1}}[\mathbf{\%}]$ & $\boldsymbol{y}_{\mathbf{2}}[\boldsymbol{\%}]$ & $\boldsymbol{y}_{\mathbf{3}}[\mathbf{\%}]$ & $\boldsymbol{y}_{\mathbf{4}}[\mathbf{\%}]$ \\
\hline 1.250 & 2.810 & 0.000 & 5.940 & 1.260 & 1.600 & 1.550 & 1.840 \\
\hline 1.350 & 5.940 & 2.810 & 17.320 & 9.000 & 8.910 & 9.070 & 8.980 \\
\hline 1.450 & 17.320 & 5.940 & 26.090 & 16.760 & 16.270 & 16.650 & 16.230 \\
\hline 1.550 & 26.090 & 17.320 & 32.490 & 24.500 & 24.270 & 24.290 & 24.230 \\
\hline 1.650 & 32.490 & 26.090 & 38.350 & 32.250 & 37.400 & 31.940 & 32.100 \\
\hline 1.750 & 38.350 & 32.490 & 48.380 & 39.990 & 40.090 & 39.490 & 39.600 \\
\hline 1.850 & 48.380 & 38.350 & 0.000 & 47.740 & 47.740 & 48.470 & 48.440 \\
\hline
\end{tabular}

Tab. 11. Ash contents by separation in accordance to particle size - coal, type 34.2

Tab. 11. Zawartość popiołu według rozdziału na klasy ziarnowe - węgiel, typ 34.2

\begin{tabular}{|c|c|c|c|c|c|c|c|}
\hline $\boldsymbol{x}_{\mathbf{1}}[\mathbf{m m}]$ & $\boldsymbol{y}[\mathbf{\%}]$ & $\boldsymbol{x}_{\mathbf{2}}[\mathbf{\%}]$ & $\boldsymbol{x}_{\mathbf{3}}[\mathbf{\%}]$ & $\boldsymbol{y}_{\mathbf{1}}[\boldsymbol{[} \%]$ & $\boldsymbol{y}_{\mathbf{2}}[\mathbf{\%}]$ & $\boldsymbol{y}_{\mathbf{3}}[\boldsymbol{\%}]$ & $\boldsymbol{y}_{\mathbf{4}}[\mathbf{[} \boldsymbol{0}]$ \\
\hline 0.750 & 7.110 & 0.000 & 8.670 & 7.600 & 6.970 & 7.480 & 6.980 \\
\hline 2.075 & 8.670 & 7.110 & 10.280 & 8.620 & 8.940 & 8.550 & 8.900 \\
\hline 4.725 & 10.280 & 8.670 & 12.950 & 10.660 & 10.840 & 10.660 & 10.820 \\
\hline 7.150 & 12.950 & 10.280 & 14.940 & 12.530 & 12.590 & 12.580 & 12.600 \\
\hline 9.000 & 14.940 & 12.950 & 15.830 & 13.960 & 14.170 & 14.030 & 14.170 \\
\hline 11.250 & 15.830 & 14.940 & 16.970 & 15.690 & 15.720 & 15.790 & 15.750 \\
\hline 13.250 & 16.970 & 15.830 & 18.480 & 17.230 & 17.230 & 17.370 & 17.270 \\
\hline 15.000 & 18.480 & 16.970 & 20.550 & 18.580 & 18.530 & 18.750 & 18.580 \\
\hline 18.000 & 20.550 & 18.480 & 0.000 & 20.890 & 20.620 & 20.530 & 20.550 \\
\hline
\end{tabular}

Tab. 12. Ash contents by separation in accordance to particle density - coal, type 34.2

Tab. 12. Zawartość popiołu według rozdziału na frakcje gęstościowe - węgiel, typ 34.2

\begin{tabular}{|c|c|c|c|c|c|c|c|}
\hline $\boldsymbol{x}_{\mathbf{1}}[\mathbf{m m}]$ & $\boldsymbol{y}[\boldsymbol{\%}]$ & $\boldsymbol{x}_{\mathbf{2}}[\mathbf{\%}]$ & $\boldsymbol{x}_{\mathbf{3}}[\mathbf{\%}]$ & $\boldsymbol{y}_{\mathbf{1}}[\mathbf{\%}]$ & $\boldsymbol{y}_{\mathbf{2}}[\boldsymbol{\%}]$ & $\boldsymbol{y}_{\mathbf{3}}[\mathbf{\%}]$ & $\boldsymbol{y}_{\mathbf{4}}[\mathbf{\%}]$ \\
\hline 1.250 & 1.270 & 0.000 & 4.280 & 0.310 & 0.210 & 0.750 & 0.640 \\
\hline 1.350 & 4.280 & 1.270 & 16.380 & 7.310 & 7.360 & 7.500 & 7.540 \\
\hline 1.450 & 16.380 & 4.280 & 24.520 & 15.090 & 15.250 & 14.660 & 14.820 \\
\hline 1.550 & 24.520 & 16.380 & 29.800 & 22.870 & 22.860 & 22.130 & 22.110 \\
\hline 1.650 & 29.800 & 24.520 & 34.790 & 30.650 & 30.580 & 29.630 & 29.560 \\
\hline 1.750 & 34.790 & 29.800 & 49.090 & 38.440 & 38.400 & 36.140 & 35.860 \\
\hline 1.850 & 49.090 & 34.790 & 0.000 & 46.220 & 46.240 & 49.300 & 49.310 \\
\hline
\end{tabular}

Tab. 13. Ash contents by separation in accordance to particle size - coal, type 35

Tab. 13. Zawartość popiołu według rozdziału na klasy ziarnowe - węgiel, typ 35

\begin{tabular}{|c|c|c|c|c|c|c|c|}
\hline $\boldsymbol{x}_{\mathbf{1}}[\mathbf{m m}]$ & $\boldsymbol{y}[\mathbf{\%}]$ & $\boldsymbol{x}_{\mathbf{2}}[\mathbf{\%}]$ & $\boldsymbol{x}_{\mathbf{3}}[\mathbf{\%}]$ & $\boldsymbol{y}_{\mathbf{1}}[\mathbf{\%}]$ & $\boldsymbol{y}_{\mathbf{2}}[\mathbf{\%}]$ & $\boldsymbol{y}_{\mathbf{3}}[\mathbf{\%}]$ & $\boldsymbol{y}_{\mathbf{4}}[\mathbf{\%}]$ \\
\hline 0.750 & 16.250 & 0.000 & 22.340 & 20.580 & 16.380 & 19.240 & 16.520 \\
\hline 2.075 & 22.340 & 16.250 & 32.180 & 23.170 & 24.320 & 22.850 & 23.910 \\
\hline 4.725 & 32.180 & 22.340 & 35.480 & 28.340 & 29.240 & 28.370 & 29.070 \\
\hline 7.150 & 35.480 & 32.180 & 37.080 & 33.060 & 35.500 & 33.280 & 35.110 \\
\hline 9.000 & 37.080 & 35.480 & 41.310 & 36.670 & 38.530 & 37.050 & 38.500 \\
\hline 11.250 & 41.310 & 37.080 & 45.300 & 41.060 & 41.220 & 42.140 & 41.770 \\
\hline 13.250 & 45.300 & 41.310 & 49.620 & 44.950 & 44.760 & 46.740 & 45.650 \\
\hline 15.000 & 49.620 & 45.300 & 50.840 & 48.360 & 47.990 & 50.050 & 48.990 \\
\hline 18.000 & 50.840 & 49.620 & 0.000 & 54.210 & 52.470 & 50.880 & 50.870 \\
\hline
\end{tabular}

ture, while factor $Z_{1}$ is related to variables $X_{1}, X_{2}, X_{3}, X_{4}$; factor $Z_{2}$ to variables $X_{2}, X_{3}, X_{4}$ and factor $Z_{3}$ to variable $X_{5}$.

Another criterion of limiting number of factors is determination of amount of percent of total variance explained by chosen factors (most often it is required to not be lower than $85 \%)$. In this case, for coal type 31 , factors $Z_{1}$ and $Z_{2}$ explain 93.14\% of variation of variable $\mathrm{X}_{1}$ (combustion heat), $96.65 \%$ of variation of variable $X_{2}$ (ash contents), $99.00 \%$ of variation of variable $X_{3}$ (sulfur contents), $91.14 \%$ of variation of variable $X_{4}$ (volatile parts contents) and $89.14 \%$ of variation of variable $X_{5}$ (analytic moisture). For coal type 34.2, factors $Z_{1}$, $Z_{2}$ and $Z_{3}$ explain $95.21 \%$ of variation of variable $X_{1}, 97.48 \%$ of variation of variable $X_{2}, 99.95 \%$ of variation of variable $X_{3}$, $86.72 \%$ of variation of variable $X_{4}$ and $99.68 \%$ of variation of variable $X_{5}$. Finally, for coal type 35 , these factors explain
$98.21 \%$ of variation of variable $X_{1}, 98.39 \%$ of variation of variable $X_{2}, 99.87 \%$ of variation of variable $X_{3}, 95.57 \%$ of variation of variable $X_{4}$ and $99.00 \%$ of variation of variable $X_{5}$.

The influences of individual factors on considered variables in all fractions of individual types of coal are presented in Tables 3-8. It was assumed that changeability of each feature should be explained by factors in at least $85 \%$.

\subsection{Mathematical modeling}

On the basis of one- and multidimensional regressive analysis four models presenting relations between ash contents in certain particle size fraction (or density fraction), particle density (or particle size) and ash contents in neighboring size or density fractions.

The general form of proposed models are: 
Tab. 14. Ash contents by separation in accordance to particle density - coal, type 35

Tab. 14. Zawartość popiołu według rozdziału na frakcje gęstościowe - węgiel, typ 35

\begin{tabular}{|c|c|c|c|c|c|c|c|}
\hline $\boldsymbol{x}_{\mathbf{1}}[\mathbf{m m}]$ & $\boldsymbol{y}[\mathbf{\%}]$ & $\boldsymbol{x}_{\mathbf{2}}[\mathbf{\%}]$ & $\boldsymbol{x}_{\mathbf{3}}[\mathbf{\%}]$ & $\boldsymbol{y}_{\mathbf{1}}[\mathbf{\%}]$ & $\boldsymbol{y}_{\mathbf{2}}[\mathbf{\%}]$ & $\boldsymbol{y}_{\mathbf{3}}[\mathbf{\%}]$ & $\boldsymbol{y}_{\mathbf{4}}[\mathbf{\%}]$ \\
\hline 1.250 & 2.370 & 0.000 & 8.290 & 0.810 & 1.770 & 0.710 & 1.710 \\
\hline 1.350 & 8.290 & 2.370 & 15.160 & 9.290 & 9.030 & 9.230 & 8.850 \\
\hline 1.450 & 15.160 & 8.290 & 27.700 & 17.770 & 17.100 & 17.810 & 17.070 \\
\hline 1.550 & 27.700 & 15.160 & 35.540 & 26.250 & 25.400 & 26.350 & 25.450 \\
\hline 1.650 & 35.540 & 27.700 & 43.360 & 34.730 & 34.980 & 34.880 & 35.550 \\
\hline 1.750 & 43.360 & 35.540 & 51.340 & 43.210 & 43.480 & 43.410 & 43.980 \\
\hline 1.850 & 51.340 & 43.360 & 0.000 & 51.690 & 51.990 & 51.370 & 51.340 \\
\hline
\end{tabular}

Tab. 15. Values of errors by particle size as partition feature

Tab. 15. Wartości błędów według wielkości ziarna jako cechy rozdziału

\begin{tabular}{|c|c|c|c|c|c|c|c|c|c|c|c|c|c|}
\hline & \multicolumn{4}{|c|}{ Coal, type 31 } & \multicolumn{4}{c|}{ Coal, type 34.2 } & \multicolumn{4}{c|}{ Coal, type 35 } \\
\cline { 2 - 14 } & $y_{1}$ & $y_{2}$ & $y_{3}$ & $y_{4}$ & & $y_{1}$ & $y_{2}$ & $y_{3}$ & $y_{4}$ & $y_{1}$ & $y_{2}$ & $y_{3}$ & $y_{4}$ \\
\hline$R^{2}$ & 0.902 & 0.919 & 0.938 & 0.941 & & 0.990 & 0.993 & 0.991 & 0.993 & 0.952 & 0.982 & 0.972 & 0.987 \\
\hline$M S E$ & 5.190 & 5.100 & 4.460 & 4.790 & & 0.498 & 0.200 & 0.490 & 0.480 & 2.760 & 1.840 & 2.260 & 1.720 \\
\hline$C p$ & 4.220 & 3.810 & 2.210 & 4.000 & & 2.540 & 2.280 & 3.490 & 4.000 & 12.290 & 3.910 & 4.480 & 4.000 \\
\hline
\end{tabular}

Tab. 16. Values of errors by particle density as partition feature Tab. 16. Wartości błędów według gęstości ziarna jako cechy rozdziału

\begin{tabular}{|c|c|c|c|c|c|c|c|c|c|c|c|c|}
\hline & \multicolumn{4}{|c|}{ Coal, type 31 } & \multicolumn{4}{c|}{ Coal, type 34.2 } & \multicolumn{4}{c|}{ Coal, type 35 } \\
\cline { 2 - 14 } & $y_{1}$ & $y_{2}$ & $y_{3}$ & $y_{4}$ & $y_{1}$ & $y_{2}$ & $y_{3}$ & $y_{4}$ & $y_{1}$ & $y_{2}$ & $y_{3}$ & $y_{4}$ \\
\hline$R^{2}$ & 0.990 & 0.990 & 0.990 & 0.991 & 0.979 & 0.979 & 0.987 & 0.988 & 0.994 & 0.996 & 0.994 & 0.995 \\
\hline$M S E$ & 1.870 & 2.060 & 2.030 & 2.310 & 2.710 & 3.020 & 2.300 & 2.610 & 1.620 & 1.620 & 1.790 & 1.770 \\
\hline$C p$ & 0.270 & 2.200 & 2.080 & 4.000 & 2.400 & 4.340 & 2.110 & 4.000 & 1.175 & 2.343 & 3.100 & 4.000 \\
\hline
\end{tabular}

- One-dimensional model

$y=a x_{1}+b$

- $\quad$ Two-dimensional models

$y=a_{1} x_{1}+a_{2} x_{2}+b$

and

$\mathrm{y}=a_{1} x_{1}+a_{2} x_{3}+b$

- Three-dimensional model

$y=a_{1} x_{1}+a_{2} x_{2}+a_{3} x_{3}+b$

where:

$y$ - ash contents in certain particle size (or particle density) fraction;

$x_{1}$ - particle size or particle density;

$x_{2}$ - ash contents in previous particle size (or density) fraction; $x_{3}$ - ash contents in following particle size (or density) fraction.

Because of the fact that during material separation process particles from other fractions transfer to the certain considered fraction in two- and three-dimensional models ash contents in neighboring fractions were taken into account and their influence was evaluated.

The analysis was conducted for all three types of coal. The results of analyzes were presented in Tables 9-14.

On the basis of the results the regressive analysis was conducted and the following models were obtained in accordance to the equations (4)-(7):

$$
\begin{aligned}
& y_{1}=2.505 x_{1}+10.910 \\
& y_{2}=1.397 x_{1}+0.385 x_{2}+10.307 \\
& y_{3}=2.407 x_{1}+0.166 x_{2}+6.480 \\
& y_{4}=1.979 x_{1}+0.153 x_{2}+0.145 x_{3}+6.720
\end{aligned}
$$

Individual models describing formulas presented in equations (4)-(7) are in this case as following:

$$
\begin{aligned}
& y_{1}=77 . .470 x_{1}-95.575 \\
& y_{2}=70.270 x_{1}+0.103 x_{2}-86.240 \\
& y_{3}=77.960 x_{1}-0.024 x_{2}-95.750 \\
& y_{4}=71.590 x_{1}+0.091 x_{2}-0.023 x_{3}-87.509
\end{aligned}
$$

In this case, functions describing relations presented in equations (4)-(7) are in form:

$\mathrm{y}_{1}=0.771 \mathrm{x}_{1}+7.022$

$\mathrm{y}_{2}=0.618 \mathrm{x}_{1}+0.162 \mathrm{x}_{2}+6.509$

$\mathrm{y}_{3}=0.770 \mathrm{x}_{1}-0.027 \mathrm{x}_{2}+6.682$

$\mathrm{y}_{4}=0.627 \mathrm{x}_{1}+0.152 \mathrm{x}_{2}-0.007 \mathrm{x}_{3}+6.453$

The individual functions describing relations given by equations (4)-(7) are in this case as following:

$$
\begin{aligned}
& y_{1}=77.822 x_{1}-97.748 \\
& y_{2}=79.838 x_{1}-0.031 x_{2}-100.381 \\
& y_{3}=80.180 x_{1}-0.105 x_{2}-99.928 \\
& y_{4}=82.123 x_{1}-0.030 x_{2}-0.105 x_{3}-101.565
\end{aligned}
$$

This time the form of the equations (4)-(7) is as following:

$$
\begin{aligned}
& y_{1}=1.949 x_{1}+19.127 \\
& y_{2}=0.895 x_{1}+0.416 x_{2}+15.706 \\
& y_{3}=1.955 x_{1}+0.104 x_{2}+15.454 \\
& y_{4}=1.118 x_{1}+0.329 x_{2}+0.057 x_{3}+14.409
\end{aligned}
$$

In this case the models created in accordance to equations (4)-(7) are in form: 
$\mathrm{y}_{1}=84.798 \mathrm{x}_{1}-105.185$

$\mathrm{y}_{2}=67.294 \mathrm{x}_{1}+0.227 \mathrm{x}_{2}-82.350$

$\mathrm{y}_{3}=84.577 \mathrm{x}_{1}+0.010 \mathrm{x}_{2}-105.096$

$\mathrm{y}_{4}=63.617 \mathrm{x}_{1}+0.268 \mathrm{x}_{2}+0.022 \mathrm{x}_{3}-77.993$

\subsection{Investigation of models quality}

To evaluate the quality of models obtained by means of general formulas presented in equations (4)-(7) such factors as $R^{2}$ coefficient, mean squared error MSE and Mallow's statistics $C_{p}$ were calculated which are given by the following formulas (Stanisz, 2007; Tumidajski and Saramak, 2009), presented in equations (8)-(10):

$R^{2}=1-\frac{\sum_{i=1}^{n}\left(y_{k i}-y_{i}\right)^{2}}{\sum_{i=1}^{u}\left(y_{i}-\bar{y}\right)^{2}}, k=1,2,3,4$

$$
M S E=\sqrt{\frac{\sum_{i=1}^{n}\left(y_{k i}-y_{i}\right)^{2}}{n-q-1}}
$$

where $q$ is an amount of independent variables occurring in considered function

$$
C_{p}=\frac{\sum_{i=1}^{n}\left(y_{k i}-y_{i}\right)^{2}}{M S E_{4}^{2}}
$$

where MSE4 is mean squared error calculated for $y_{4}$.

The obtained results of calculated errors are presented in Tables 15 and 16.

\section{Conclusions}

Because of the fact that the most often three factors occur in individual fractions and considering power of relations between individual properties the investigated variables can be divided into three subsets. First one contains combustion heat, ash contents and volatile parts contents, second one contains sulfur contents and the third one contains moisture. In scientific works $[3,4,5,6,7,8,9,12,13,14,15,16,17,18$, $19,20,23,24]$, through application of various visualization methods it was claimed that features being sufficient to identify coal type are sulfur contents, moisture and volatile parts contents. The conducted analysis confirms these results. The selection of variable $X_{4}$ (volatile parts contents) occurs from the fact that this variable is explained by other factor than mutual factor with variables moisture and combustion heat.

Considering the mathematical models it must be said that during grained material separation (in this case - coal) into particle size or density fractions some of the particles from neighboring fractions $(j-1$ or $j+1)$ occur in $j$ th fraction it seems to be justified to consider this fact during construction of mathematical model describing ash contents by means of particle size or density.

In the paper four models are proposed:

- One-dimensional, which does not consider influences of neighboring fractions;

- Two-dimensional, which takes the influence of one of neighboring fractions into consideration - two models of such type;

- Three-dimensional, which takes the influence of both neighboring fractions.

The verification of these models was conducted on the basis of three factors: $R^{2}$ coefficient, mean squared error MSEand Mallow's statistics $C_{p}$.

Taking into consideration the $R^{2}$ coefficient it is visible that for all considered models the value of this factor is relatively high (above 0.9). It can be noticed that the $R^{2}$ achieves higher values when the separation is done in accordance to particle density than in case of particle size (apart from coal, type 34.2).

Furthermore, the value of mean squared error indicates that the models are well fitted, but (apart from coal, type 34.2) significantly better fitting to empirical results is achieved in case of separation done in accordance to particle density. To compare the models for various dimensions the Mallow's statistics $C_{p}$ was used, which suggests that the best model is the one which values of $C_{p}$ is close to the value $q+1$, where $\mathrm{q}$ is a number of independent variables occurring in the model. Analyzing Tables 8 and 9 it can be stated that the best model is a three-dimensional one, but in some cases, as for coal, type 35 by separation done in accordance to particle size, the two-dimensional models have the value of $C_{p}$ around $q+1=3$.

The analyzed cases indicate that despite satisfying results of one-dimensional approximation to obtain better models is worthy to consider also influences of the researched feature in neighboring fractions.

\section{Acknowledgement}

The paper is a result of AGH UST statuory project no. 11.11.100.276. 


\section{Literatura - References}

1. BROŻEK M. Evaluation of liberation level of coal mineral fraction on the basis of Hall's separation curve, Mining Review, 11, pp. 384-387, 1984. (in Polish)

2. COMREY A. L. A first Course in Factor Analysis New York Academic Press, 1973.

3. DOBOSZ M. Statistical analysis of research results, Akademicka Oficyna Wydawnicza Exit Warsaw, 2001. [in Polish]

4. FOSZCZ D., DUCHNOWSKA M., NIEDOBA T., TUMIDAJSKI T. Accuracy of separation parameters resulting errors of chemical analysis, experimental results and data approximation, Physicochemical Problems of Mineral Processing, 52(1), pp. 98-111, 2016.

5. FOSZCZ D., NIEDOBA T., TUMIDAJSKI T. A geometric approach to evaluating the results of Polish copper ores beneficiation, Mineral Resources Management; 34(2), pp. 55-66, 2018.

6. GEOWIAK S. The reasons for necessity to correct some part of gravitational enrichment theory, Journal of the Polish Mineral Engineering Society, 1(43), pp. 199-210, 2019a.

7. GŁOWIAK S. Assumptions of probabilistic model of grains density distribution in jig bed layers, Journal of the Polish Mineral Engineering Society, 1(43), pp. 211-220, $2019 \mathrm{~b}$.

8. JAMRÓZ D. Application of Multidimensional Data Visualization in Creation of Pattern Recognition Systems, In: Gruca A., Czachórski T., Kozielski S. (eds.), Man-Machine, Interactions 3 AISC Switzerland Springer International Publishing, 242, pp. 443-450, 2014a.

9. JAMRÓZ D. Application of multidimensional scaling to classification of various types of coal, Archives of Mining Sciences, 59(2), pp. 413-425, 2014b.

10. JAMRÓZ D. Application of multi-parameter data visualization by means of autoassociative neural networks to evaluate classification possibilities of various coal types, Physicochemical Problems of Mineral Processing, 50(2), pp. 719-734, 2014c.

11. JAMRÓZ D. Multidimensional labyrinth - multidimensional virtual reality. In: Cyran K., Kozielski S., Peters J., Stanczyk U., Wakulicz-Deja A. (eds.): Man-Machine, Interactions, AISC Heidelberg Springer-Verlag, 59, pp. 445-450, 2009.

12. JAMRÓZ D., NIEDOBA T. Application of multidimensional data visualization by means of self-organizing Kohonen maps to evaluate classification possibilities of various coal types, Archives of Mining Sciences, 60(1), pp. 39-50, 2015a.

13. JAMRÓZ D., NIEDOBA T. Application of Observational Tunnels Method to Select Set of Features Sufficient to Identify a Type of Coal, Physicochemical Problems of Mineral Processing, 50(1), pp. 185-202, 2014.

14. JAMRÓZ D., NIEDOBA T. Comparison of selected methods of multi-parameter data visualization used for classification of coals, Physicochemical Problems of Mineral Processing, 51(2), pp. 769-784, 2015b.

15. JAMRÓZ D., NIEDOBA T., SUROWIAK A., TUMIDAJSKI T. The use of the visualization of multidimensional data using PCA to evaluate possibilities of the division of coal samples space due to their suitability for fluidised gasification, Archives of Mining Sciences, 61(3), pp. 523-535,. 2016.

16. JAMRÓZ D., NIEDOBA T., SUROWIAK A., TUMIDAJSKI T., SZOSTEK R., GAJER M. Application of multi-parameter data visualization by means of multidimensional scaling to evaluate possibility of coal gasification, Archives of Mining Sciences, 62(3), pp. 445-457, 2017.

17. KLINE P. An easy Guide to Factor Analysis Routledge London, 1994.

18. LAWLEY D.N., MAXWELL A.E. Factor Analysis as a Statistical Method London Butterworths, 1971.

19. NIEDOBA T. Application of Relevance Maps in Multidimensional Classification of Coal Types, Archives of Mining Sciences, 60(1), pp. 93-106, 2015.

20. NIEDOBA T. JAMRÓZ D. Visualization of multidimensional data in purpose of qualitative classification of various types of coal, Archives of Mining Sciences, 58(4), pp. 1317-1333, 2013.

21. NIEDOBA T. Multidimensional characteristics of random variables in description of grained materials and their separation processes, Wydawnictwo Instytutu Gospodarki Surowcami Mineralnymi i Energią PAN Kraków, 2013a [in Polish].

22. NIEDOBA T. Multidimensional distributions of grained materials characteristics by means of non-parametric approximation of marginal statistical density function, AGH Journal of Mining and Geoengineering, 4, pp. 235-244, 2009. [in Polish].

23. NIEDOBA T. Multi-parameter data visualization by means of principal component analysis (PCA) in qualitative evaluation of various coal types, Physicochemical Problems of Mineral Processing, 50(2), pp. 575-589, 2014. 
24. NIEDOBA T. Statistical analysis of the relationship between particle size and particle density of raw coal, Physicochemical Problems of Mineral Processing, 49(1), pp. 175-188, $2013 \mathrm{~b}$.

25. NIEDOBA T. Three-dimensional distribution of grained materials characteristics, in Proceedings of the XIV Balkan Mineral Processing Congress Tuzla Bosnia and Herzegovina, 1, pp. 57-59, 2011.

26. NIEDOBA T., PIĘTA P., SUROWIAK A. Analysis of distributions of various coal types properties by means of statistical methods IOP Conference Series: Materials Science and Engineering art. 012008, 427, pp. 1-5, 2018.

27. NIEDOBA T., SUROWIAK A. Type of coal and multidimensional description of its composition with density and ash contents taken into consideration, in Proceedings of the XXVI International Mineral Processing Congress, 1, pp. 3844-3854, 2012.

28. ÖNEY Ö., The Increase Of The Performance Of Ultrafine Coal Flotation By Using Emulsified Kerosene And The Prediction Of The Flotation Parameters By Random Forest And Genetic Algorithm, Archives of Mining Sciences, 64(1), pp. 119-130, 2019.

29. PIĘTA P., NIEDOBA T., SUROWIAK A., ŞAHBAZ O., KARAGÜZEL C., CANIEREN Ö. Studies on Polish copper ore beneficiation in Jameson cell, IOP Conference Series: Materials Science and Engineering, 427, art. 012009, pp. $1-12,2018$.

30. SOBOLEWSKI A., MICOREK T., WINNICKA G., HEILPERN S. Proposal of Polish Coking Coal Classification, The Polish Mining Review, 72(10), pp. 38-43, 2016. [in Polish]

31. STANISZ A. Easy Course of Statistics, vol. 3, Statsoft Krakow Poland, 2007. [in Polish]

32. STĘPIŃSKI W. Mean values curves, Ores and Non-Ferrous Metals, 9(10), pp. 532-535, 1965. (in Polish)

33. SUROWIAK A. Influence of particle density distributions of their settling velocity for narrow size fractions, Mineral Resources Management, 30, pp. 105-122, 2014. [in Polish]

34. SUROWIAK A. Investigation of hard coal beneficiation destined to gasification process in fluidized bed gas generator, The Polish Mining Review, 69(2), pp. 239-244, 2007. [in Polish]

35. TUMIDAJSKI T., SARAMAK D. Methods and models of mathematical statistics in mineral processing, Wydawnictwo AGH, Krakow, 2009. (in Polish)

\begin{abstract}
Analiza czynnikowa i modelowanie matematyczne w określeniu jakości wegla
Wykonano rozdział trzech typów węgla o różnych charakterystykach, pochodzących z trzech różnych kopalni węgla kamiennego w Polsce (typy 31, 34.2 oraz 35, według Polskich norm, którymi były węgiel energetycznym, pót-koksujaccy oraz koksujący) na klasy ziarnowe a następnie na frakcje gęstościowe. Dla każdej otrzymanej w ten sposób frakcji wielkościowo-gęstościowej zmierzono następujące parametry: ciepło spalania, zawartość popiołu, zawartość siarki, zawartość części lotnych, wilgotność analityczna. W ten sposób otrzymano siedmiowymiarowy wektor danych. Za pomoca analizy czynnikowej wybrano istotne cechy wegla, które decyduja o jego przynależności do określonego typu węla. Aby ocenić prawidłowość zastosowanej metody wykorzystano test sferyczności Bartletta oraz wspótczynnik Kaisera-Mayera-Olkina (KMO). Otrzymane wyniki porównano z wynikami otrzymanymi w poprzednich pracach, które uzyskano metodą tuneli obserwacyjnych. Wyniki pokazały, które cechy węgla są niezbędne do określenia typu wegla, co wpływa na dobór odpowiedniej metody jego wzbogacania. Ponadto, zaprezentowano model prezentujacy relacje pomiędzy tymi cechami a wielkością i gęstościq ziaren. Ponieważ ziarna określonej wielkości lub gęstości moga występować w sąsiednich klasach lub frakcjach, wykonano trzy typy modeli, bazując na analizie regresji. Analiza została wykonana dla trzech typów wegli. Ponieważ modele zawierają różne ilości zmiennych niezależnych do oceny i porównania otrzymanych wyników zastosowano współczynnik determinacji $R^{2}$, błąd średniokwadratowy (MSE) oraz statystykę Mallowa $C_{\dot{p}}$.
\end{abstract}

Słowa klucze: wegiel, wielowymiarowa analiza statystyczna, analiza czynnikowa, jakość węgla, wielkość ziarna, gęstość ziarna 